\title{
Rapid Temperature-Programmed Separation of Carbon Monoxide and Carbon Dioxide on a Packed Capillary Column in Gas Chromatography: Application to the Evaluation of Photocatalytic Activity of $\mathrm{TiO}_{2}$
}

\author{
Ikuo Ueta, ${ }^{* \dagger}$ Ayako MizuguchI,* Kazue TanI, ${ }^{*}$ Susumu Kawakubo, ${ }^{*}$ and Yoshihiro SaIto** \\ *Department of Applied Chemistry, University of Yamanashi, Kofu 400-8511, Japan \\ **Department of Environmental and Life Sciences, Toyohashi University of Technology, Toyohashi 441-8580, \\ Japan
}

\begin{abstract}
Carbon monoxide $(\mathrm{CO})$ and carbon dioxide $\left(\mathrm{CO}_{2}\right)$ were determined in a conventional capillary gas chromatography-flame ionization detector (GC-FID) by introducing a packed-capillary column and a methanizer. Due to good compatibility with rapid temperature-programmed operation of the packed capillary column, several volatile compounds, including $\mathrm{CO}$ and $\mathrm{CO}_{2}$, were rapidly eluted along with satisfactory resolution and sample loading capacity. The limit of quantifications of $\mathrm{CO}$ and $\mathrm{CO}_{2}$ were 5 and $3 \mathrm{ppm}$, respectively, with an injection volume of $0.5 \mathrm{~mL}$. The developed system was then successfully applied to evaluating the photocatalytic decomposition of volatile organic compounds on titanium dioxide $\left(\mathrm{TiO}_{2}\right)$.
\end{abstract}

(Received February 14, 2013; Accepted April 13, 2013; Published June 10, 2013)

\section{Introduction}

Accurate and sensitive determinations of carbon monoxide (CO) and carbon dioxide $\left(\mathrm{CO}_{2}\right)$ are important for several research fields, such as environmental science, ${ }^{1}$ biochemistry ${ }^{2}$ and photocatalytic chemistry. ${ }^{3}$ Several analytical methods have been developed for the determination of $\mathrm{CO}$ and $\mathrm{CO}_{2}$. Infrared (IR) detectors $^{4}$ and electrochemical sensors $^{5}$ are suitable for continuous measurements of these compounds; however, some of them are interfered by other gaseous compounds. In addition, these techniques are not suitable for the analysis of small amounts of gaseous samples.

Gas chromatographic (GC) methods have been employed for simultaneous determinations of complex mixtures. ${ }^{6,7}$ For the determination of inorganic gases, a thermal conductivity detector (TCD) is widely used; however, it is not suitable for the sensitive determinations, because the TCD is not able to detect concentrations below a few hundred ppm. In the GC method, a methanation reaction by a methanizer has usually been used for sensitive determinations of $\mathrm{CO}$ and $\mathrm{CO}_{2}$ before flame ionization detection (FID). ${ }^{7}$ By introducing this technique, $\mathrm{CO}$ and $\mathrm{CO}_{2}$ can be successfully detected at few ppm levels. In typical GC analysis, adequate sensitivities of target compounds are easily obtained, even if the sample injection volume is relatively small.

Complete separation of $\mathrm{CO}$ and $\mathrm{CO}_{2}$ is necessary for accurate and precise determination in GC. In order to separate these permanent gases, a packed column has been typically used, because of its unique selectivity and retentivity as a GC stationary phase. In addition, a typical methanation reactor has been designed for connection to a conventional packed column

† To whom correspondence should be addressed.

E-mail: iueta@yamanashi.ac.jp having a typical internal diameter of 3-4 mm. However, in recent GC analysis, a capillary GC system equipped with opentubular capillary columns has been widely used for the determination of volatile organic compounds (VOCs) in samples.

Our research group has developed novel packed-capillary columns for the determination of several complex mixtures in a conventional capillary GC system. ${ }^{8}$ In these columns, a particulate stationary phase is packed into a thin-wall stainless-steel capillary of $1.0 \mathrm{~mm}$ i.d.; two stainless-steel capillaries of $0.52 \mathrm{~mm}$ o.d. are attached to the inlet and outlet of the column. Therefore, the developed column can be installed in a conventional capillary GC system without any modifications or adapters. Rapid temperature-programmed separation ${ }^{8}$ and heart-cutting analysis of complex sample mixtures ${ }^{9}$ have been investigated using the developed column.

In this study, $\mathrm{CO}$ and $\mathrm{CO}_{2}$ were successfully determined in a conventional capillary GC-FID system based on the introduction of a packed-capillary column and a methanizer. After an evaluation of the developed system performance, the method was applied to an evaluation of the photocatalytic properties of titanium dioxide $\left(\mathrm{TiO}_{2}\right)$ for typical gaseous VOCs.

\section{Experimental}

\section{Chemicals and materials}

Standard gas of $\mathrm{CO}, \mathrm{CO}_{2}$ and alkanes from $\mathrm{C}_{1}$ to $\mathrm{C}_{4}$ were purchased from GL Sciences (Tokyo, Japan). Alkanes from $\mathrm{C}_{5}$ to $\mathrm{C}_{10}$, benzene and toluene, were obtained from Tokyo Kasei Kogyo (Tokyo, Japan). Isopropoxy titanium and 2-propanol were obtained from Nacalai Tesque (Kyoto, Japan). Hydrochloric acid $(\mathrm{HCl})$ and glycerol were obtained from Kanto Chemical (Tokyo, Japan). 


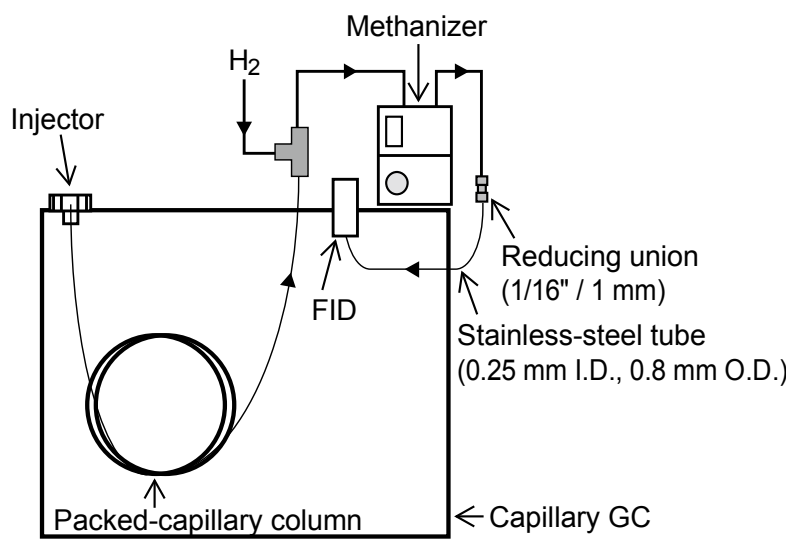

Fig. 1 Schematic illustration of the capillary GC system equipped with a packed-capillary column and a methanizer.

\section{GC-measurements}

GC-FID analyses were conducted using two Shimadzu GC-2010 gas chromatographs (Kyoto, Japan). The injection temperature and FID temperature were set at $250^{\circ} \mathrm{C}$, and all of the injections were operated in a split mode with a ratio of 5:1. As the carrier gas, He at a head pressure of 200 and $150 \mathrm{kPa}$ was used for packed-capillary column separation and fused-silica capillary separation, respectively. An MTN-1 methanizer (Shimadzu, Japan) was used for the determination of CO and $\mathrm{CO}_{2}$. An HR-1 fused-silica capillary column, $25 \mathrm{~m} \times 0.25 \mathrm{~mm}$ with a $0.25-\mu \mathrm{m}$ film thickness (Shinwa Chemical Industries, Kyoto, Japan), was used to measure alkanes from $\mathrm{C}_{5}$ to $\mathrm{C}_{10}$, benzene and toluene.

The gas samples of $\mathrm{CO}, \mathrm{CO}_{2}$ and alkanes from $\mathrm{C}_{1}$ to $\mathrm{C}_{4}$ were separated using a packed-capillary column. The column was prepared by the same procedure as described previously. ${ }^{8}$ A stationary phase of Sunpak-A (Shinwa Chemical Industries) particles of between 100 and 80 mesh, corresponding to about 150 and $180 \mu \mathrm{m}$ in diameter, was packed into a stainless-steel capillary of $1.0 \mathrm{~mm}$ i.d., $1.27 \mathrm{~mm}$ o.d., $1.0 \mathrm{~m}$ length. Then, two stainless-steel capillaries of $0.3 \mathrm{~mm}$ i.d., $0.52 \mathrm{~mm}$ o.d., $0.5 \mathrm{~m}$ length were attached to the inlet and outlet of the packed-capillary column for easy installation to a conventional GC injector and detector designed for the typical capillary column connection. The capillary GC system equipped with a packed-capillary column and a methanizer used in this study is illustrated in Fig. 1. The column outlet was connected to a tee-connector, and both of the separated $\mathrm{CO}$ and $\mathrm{CO}_{2}$ were converted to $\mathrm{CH}_{4}$, in a heated reactor tube packed with a Ni catalyst. The outlet of the methanizer was connected to a FID using a reducing union and a stainless-steel tube.

\section{Preparation of $\mathrm{TiO}_{2}$ particles for photo catalysts}

$\mathrm{TiO}_{2}$ particles were prepared based on a previous study, ${ }^{10}$ described as follows. First, $30 \mathrm{~mL}$ of a $6.0 \times 10^{-5} \mathrm{M} \mathrm{HCl}$ solution, $1500 \mathrm{~g}$ of 2-propanol and $900 \mathrm{~g}$ of glycerol were placed in a beaker and mixed for 5 min under ultrasonication. Then, $60 \mathrm{~mL}$ of an isopropoxy titanium solution was dropped into the solution under magnetic stirring, and the solution was left standing in the dark at room temperature for 4 days. The supernatant liquid was then transferred to another beaker, and stored again for 7 days. The obtained precipitation was washed with pure water, and then a classification of particle size was also made. The particles were then washed with methanol, and finally heated for $24 \mathrm{~h}$ at $200^{\circ} \mathrm{C}$. The obtained particles were
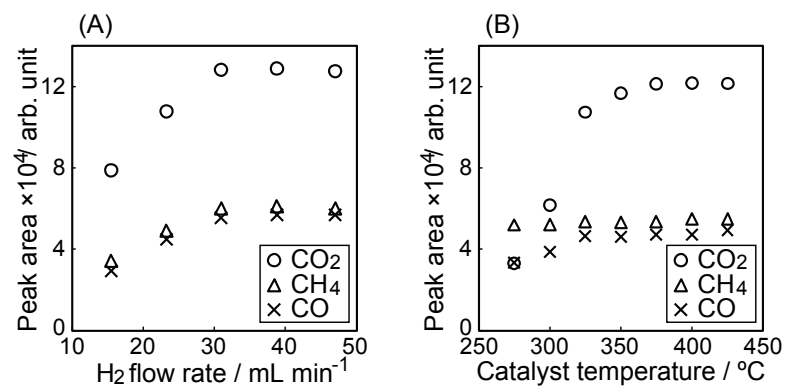

Fig. 2 Optimization of (A) $\mathrm{H}_{2}$ flow rate and (B) methanation temperature for the methanation conditions of $\mathrm{CO}$ and $\mathrm{CO}_{2}$ Conditions: sample concentration, $100 \mathrm{ppm}$ each; injection volume, $0.5 \mathrm{~mL}$; methanation temperature, (A) $440^{\circ} \mathrm{C} ; \mathrm{H}_{2}$ flow rate, (B) $40 \mathrm{~mL} / \mathrm{min}$

Table 1 LODs and LOQs for $\mathrm{CO}$ and $\mathrm{CO}_{2}$

\begin{tabular}{ccc}
\hline & $\mathrm{CO}$ & $\mathrm{CO}_{2}$ \\
\hline LOD, ppm & 2.0 & 1.0 \\
LOQ, ppm & 5.0 & 3.0 \\
\hline
\end{tabular}

Injection volume: $0.5 \mathrm{~mL}$.

porous spherical particles having $3-4 \mu \mathrm{m}$ diameter, and the specific surface area was approximately $90 \mathrm{~m}^{2} / \mathrm{g}$.

\section{Results and Discussion}

\section{Construction of a novel $\mathrm{CO}$ and $\mathrm{CO}_{2}$ detection system}

First, the methanation conditions of standard $\mathrm{CO}$ and $\mathrm{CO}_{2}$ gas were optimized. $\mathrm{CH}_{4}$ was also used as a reference. The flow rate of $\mathrm{H}_{2}$ gas supplied from a tee-connector was investigated in the range from 10 to $50 \mathrm{~mL} / \mathrm{min}$. During this time, the flow rate of $\mathrm{H}_{2}$ gas to FID was fixed at $10 \mathrm{~mL} / \mathrm{min}$. The rate was optimized by the obtained peak area of both $\mathrm{CO}$ and $\mathrm{CO}_{2}$. As shown in Fig. 2A, the methanation reaction reached a plateau at more than $30 \mathrm{~mL} / \mathrm{min}$ of $\mathrm{H}_{2}$ gas. The smaller peak areas at a low $\mathrm{H}_{2}$ flow rate could be due to a sensitivity change of FID that depended on the introduced $\mathrm{H}_{2}$ volume. Therefore, $40 \mathrm{~mL} / \mathrm{min}$ of $\mathrm{H}_{2}$ gas was employed in following experiments. The temperature of the reaction tube packed with the Ni catalyst was also investigated. The maximum peak areas were obtained at above $400^{\circ} \mathrm{C}$, as demonstrated in Fig. $2 \mathrm{~B}$; hence, a catalyst temperature of $440^{\circ} \mathrm{C}$ was chosen in this study. In Fig. 2, peak area of $\mathrm{CO}_{2}$ is greater than that of $\mathrm{CO}$. This is because a greater amount of $\mathrm{CO}_{2}$ was included as an impurity in $\mathrm{N}_{2}$ gas that was used for preparing standard gas samples.

The limit of detection (LOD) and the limit of quantification (LOQ) for $\mathrm{CO}$ and $\mathrm{CO}_{2}$ were investigated under these optimized conditions. The results are tabulated in Table 1. The LOD of the method was identified to have a signal-to-noise ratio of 3 , and the LOQ was determined to have a ratio of 10. As expected, the sensitivities for these compounds using the developed system are almost the same as that obtained by using a conventional packed column, because the number of theoretical plates for the packed-capillary column and a conventional packed column are the same. The upper LOQs for $\mathrm{CO}$ and $\mathrm{CO}_{2}$ were more than $10 \%$, and linear calibration curves were obtained with correlation coefficients greater than 0.99 for both 
compounds from the LOQ to the upper LOQ. As described in a previous publication, ${ }^{8}$ the relative delay of the retention time from the theoretically estimated value was less than $4.6 \%$ at a temperature programmed rate of $40^{\circ} \mathrm{C} / \mathrm{min}$, using the packedcapillary column. A typical chromatogram for the determinations of $\mathrm{CO}, \mathrm{CO}_{2}$ and low molecular weight alkanes is shown in Fig. 3. Successful separation and determination of a mixture of

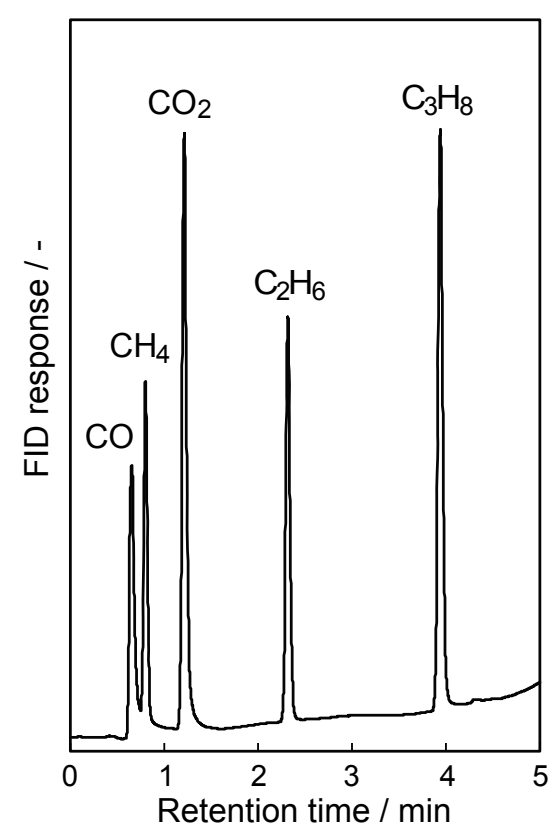

Fig. 3 Typical chromatogram for the separation of volatile compounds. Conditions: column temperature, $40^{\circ} \mathrm{C}(1 \mathrm{~min})$ to $200^{\circ} \mathrm{C}$ at $40^{\circ} \mathrm{C} / \mathrm{min}$; sample concentration, $100 \mathrm{ppm}$ each; injection volume, $0.1 \mathrm{~mL}$. volatile compounds was obtained in the developed system with a rapid temperature program of $40^{\circ} \mathrm{C} / \mathrm{min}$ without any significant peak broadening problem. In addition, no peak distortion was observed because of a high peak loading capacity of the packedcapillary column. The RSDs of the peak area for both compounds for a standard gaseous sample $(500 \mathrm{ppm})$ were lower than $5.0 \%(n=5)$.

Evaluation of the photocatalytic decomposition of $\mathrm{VOCs}$ on $\mathrm{TiO}_{2}$

On the basis of the above-mentioned rapid and sensitive determination of $\mathrm{CO}$ and $\mathrm{CO}_{2}$, the developed system was applied to an evaluation of the photocatalytic degradation of VOCs on $\mathrm{TiO}_{2}$ particles. As the VOCs sample, alkanes of $\mathrm{C}_{1}$ to $\mathrm{C}_{10}$, benzene and toluene, were investigated. First, $0.3 \mathrm{~g}$ of synthesized $\mathrm{TiO}_{2}$ particles were placed in a quartz test tube (12 cm length, $9.5 \mathrm{~mm}$ i.d.), and the test tube was closed by a silicon septum. The test tube was held horizontally, where the surface area of the $\mathrm{TiO}_{2}$ particles (irradiation area of ultraviolet (UV)) was kept to constant. After loading VOC sample into the test tube, the peak area of the added $\mathrm{VOC}$ and $\mathrm{CO}_{2}$ was measured at every $15 \mathrm{~min}$ for $60 \mathrm{~min}$. Then, UV light $(365 \mathrm{~nm})$ was irradiated to the test tube. The results are given in Fig. 4, where the variations in the amounts of the representative VOCs loaded into the test tube are plotted along with that of $\mathrm{CO}_{2}$, indicating significant adsorptions of all the VOCs onto $\mathrm{TiO}_{2}$ after the injection of gaseous VOCs into the test tube. The amount of $\mathrm{CO}_{2}$ remarkably increased upon the irradiation of UV light; at the same time, the VOCs also clearly decreased due to UV irradiation for all of the investigated compounds by photocatalitic oxidation on $\mathrm{TiO}_{2}$. The amounts of the loaded VOCs did not agree with the amount of the increased $\mathrm{CO}_{2}$. This is because the generated $\mathrm{CO}_{2}$ was also adsorbed onto the $\mathrm{TiO}_{2}$ particles. Typically, the photocatalytic property of a catalyst has been evaluated by using a flow-type reactor. ${ }^{11-13}$ However, for evaluating several properties of a catalyst, such as the adsorption of VOCs on the catalyst, and determining the photocatalytic
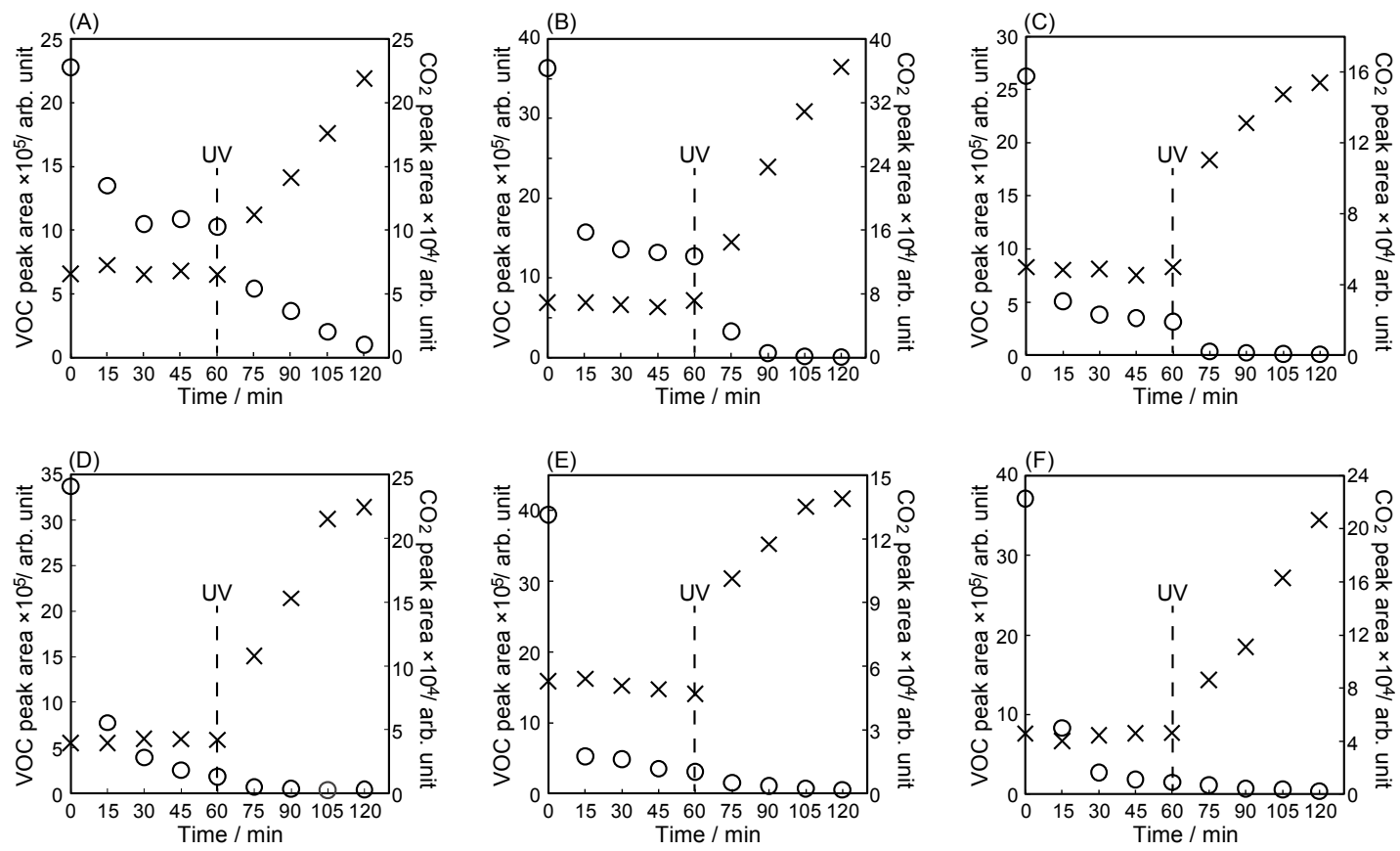

Fig. 4 Investigation of the photocatalytic degradation of $\mathrm{VOCs}$ on $\mathrm{TiO}_{2}$ particles by the determination of $\mathrm{CO}_{2}$ (cross) and VOCs (circle). Investigated VOCs: (A) ethane, (B) butane, (C) hexane, (D) octane, (E) decane, $(\mathrm{F})$ toluene. Sample injection volume, $0.1 \mathrm{~mL}$. 
rate, the determination of both the $\mathrm{VOC}$ and $\mathrm{CO}_{2}$ concentrations in a closed system could also be effective. Because the developed method requires only a small amount of a gaseous sample, it could be suitable for evaluating the photocatalytic property of a catalyst in a closed system.

\section{Conclusions}

A sensitive and rapid determination method for $\mathrm{CO}$ and $\mathrm{CO}_{2}$ in a conventional capillary GC system was developed by introducing a packed-capillary column and a methanizer without any significant system modification. There are many potential requirements for an easy determination of $\mathrm{CO}$ or $\mathrm{CO}_{2}$ with a small amount of the sample volume in many research fields; therefore, further applications of the method could be expected: such as the determination of atmospheric gas and combustion gas samples. The proposed method was also successfully applied to evaluating the photocatalytic property of $\mathrm{TiO}_{2}$ particles for typical gaseous VOCs. The results indicated that the developed technique could be a relatively easy evaluation method of the photocatalytic property of catalysts. The method could also be suitable for this kind of the evaluation of several catalytic properties in a capillary GC system.

\section{Acknowledgements}

Part of this research was financially supported by a Grant-inAid for Scientific Research from the Japan Society for the Promotion of Science (JSPS). The authors acknowledge the technical support of Prof. H. Okuzaki, Prof. N. Miyajima and H. Koizumi of University of Yamanashi.

\section{References}

1. W. Soon, S. L. Baliunas, A. B. Robinson, and Z. W. Robinson, Clim. Res., 1999, 13, 149.

2. L. Keck, C. Houechen, and U. Oeh, Int. J. Mass Spectrom., 2008, 270, 156.

3. A. Fujishima, X. Zhang, and D. A. Tryk, Surf. Sci. Rep., 2008, 63, 515.

4. M. B. Esler, D. W. T. Griffith, S. R. Wilson, and L. P. Steele, Anal. Chem., 2000, 72, 206.

5. M. Ando, Trends Anal. Chem., 2006, 25, 937.

6. J. B. Laurens, J. P. de Coning, and J. M. Swinley, J. Chromatogr., A, 2001, 911, 107.

7. M. Kamiński, R. Katranowica, D. Jastrzębski, and M. M. Kamiński, J. Chromatogr., A, 2003, 989, 277.

8. M. Inoue, Y. Saito, I. Ueta, T. Miura, H. Ohkita, K. Fujimura, and K. Jinno, Anal. Sci., 2010, 26, 687.

9. I. Ueta, K. Takahashi, and Y. Saito, Anal. Sci., 2012, 28, 953.

10. K. Tani and Y. Suzuki, J. Chromatogr., A, 1996, 722, 129.

11. C. T. Brigden, S. Poulston, M. V. Twigg, A. P. Walker, and A. J. J. Wilkins, Appl. Catal., B, 2001, 32, 63.

12. A. K. Boulamanti and C. J. Philippopoulos, Atmos. Environ., 2009, 43, 3168.

13. M. Hussain, N. Russo, and G. Saracco, Chem. Eng. J., 2011, 166, 138. 